Proceedings of the

International Geometry Center

Vol. 12, no. 2 (2019) pp. 11-25

\title{
A $(\mathrm{CHR})_{3}$-flat trans-Sasakian manifold
}

\author{
Koji Matsumoto
}

\begin{abstract}
In [4] M. Prvanovic considered several curvaturelike tensors defined for Hermitian manifolds. Developing her ideas in [3], we defined in an almost contact Riemannian manifold another new curvaturelike tensor field, which is called a contact holomorphic Riemannian curvature tensor or briefly $(\mathrm{CHR})_{3}$-curvature tensor. Then, we mainly researched $(\mathrm{CHR})_{3}$-curvature tensor in a Sasakian manifold. Also we proved, that a conformally $(\mathrm{CHR})_{3^{-}}$ flat Sasakian manifold does not exist.

In the present paper, we consider this tensor field in a trans-Sasakian manifold. We calculate the $(\mathrm{CHR})_{3}$-curvature tensor in a trans-Sasakian manifold. Also, the $(\mathrm{CHR})_{3}$-Ricci tensor $\rho_{3}$ and the $(\mathrm{CHR})_{3}$-scalar curvature $\tau_{3}$ in a trans-Sasakian manifold have been obtained.

Moreover, we define the notion of the $(\mathrm{CHR})_{3}$-flatness in an almost contact Riemannian manifold. Then, we consider this notion in a trans-Sasakian manifold and determine the curvature tensor, the Ricci tensor and the scalar curvature. We proved that a $(\mathrm{CHR})_{3}$-flat trans-Sasakian manifold is a generalized $\eta$-Einstein manifold.

Finally, we obtain the expression of the curvature tensor with respect to the Riemannian metric $g$ of a trans-Sasakian manifold, if the latter is $(\mathrm{CHR})_{3}$-flat.
\end{abstract}

Анотація. У [4] М. Прванович розглянуто декілька кривиноподібних тензорів, визначених для ермітових різновидів. Розвиваючи їі ідеї у [3], ми ввводимо в майже контактному рімановому многовиді ще одне нове кривиноподібне тензорне поле, яке називається тензором контактної голоморфної ріманової кривини або коротко тензором $(C H R)_{3}$-кривини. Далі ми, головним чином, досліджуємо тензор $(C H R)_{3}$-кривини сасакійового многовиду. Крім того, ми доводимо, що конформно $(C H R)_{3}$ плоского сасакійового многовиду не існує.

$\mathrm{У}$ цій роботі ми розглядаємо це тензорне поле на транссасакійовому многовиді. Нами обчислено тензор $(C H R)_{3}$-кривини транссасакійового многовиду. Також були отримані $(C H R)_{3}$-Річчі тензор $\rho_{3}$ та $(C H R)_{3}$ скалярна кривина $\tau_{3}$ транссасакійового многовиду.

Keywords: $(\mathrm{CHR})_{3}$-curvature tensor, trans-Sasakian manifold, $(\mathrm{CHR})_{3}$-flat almost contact Riemannian manifold, (generalized) $\eta$-Einstein manifold.

DOI: http://dx.doi.org/10.15673/tmgc.v12i2.1438 
Крім того, ми вводимо поняття $(C H R)_{3}$-плоских майже контактних ріманових многовидів. Нами розглянуто $(C H R)_{3}$-плоскі транс-сасакійові многовиди, для яких ми знаходимо тензор кривини, тензор Річчі та скалярну кривину. Нами доведено, що $(C H R)_{3}$-плоский транссасакійовий многовид є узагальненим $\eta$-ейнштейновим многовидом.

Нарешті, нами отримно вирази тензору кривини ріманової метрики $g$ транссасакійового многовиду, якщо останній є $(C H R)_{3}$-плоским.

\section{Almost contact Riemannian manifolds}

A real $(2 n+1)$-dimensional differentiable Riemannian manifold $\left(M^{2 n+1}, g\right)$ is said to be an almost contact Riemannian manifold if it has a $(1,1)$-tensor $\varphi$ and a 1-form $\eta$ which satisfy

$$
\begin{gathered}
\varphi^{2}=-I+\eta \otimes \xi, \quad \eta(\varphi X)=0, \quad \eta(\xi)=1, \\
g(\varphi X, \varphi Y)=g(X, Y)-\eta(X) \eta(Y)
\end{gathered}
$$

for any $Y, X \in T M^{2 n+1}$, where $\xi$ is defined by

$$
g(\xi, X)=\eta(X)
$$

and $T M^{2 n+1}$ is the tangent bundle of $M^{2 n+1}$.

From $(1.1)_{3}$, the vector field $\xi$ is unit and we call this vector field the structure vector field of the almost contact Riemannian manifold. Next, in an almost contact Riemannian manifold $M^{2 n+1}$ we define a 2-form $F$ by

$$
F(X, Y)=g(\varphi X, Y)
$$

for all $X, Y \in T M^{2 n+1}$. Then the 2-form $F$ is skew-symmetric and we call this tensor field the fundamental 2-form of this almost contact Riemannian manifold. Hereafter, we write the same $\varphi$ instead of $F$.

An almost contact manifold $M^{2 n+1}$ is called trans-Sasakian if the fundamental form $\varphi$ satisfies

$$
\begin{aligned}
\left(\nabla_{X} \varphi\right)(Y, Z)=\alpha & \{g(X, Y) \eta(Z)-g(X, Z) \eta(Y)\}+ \\
& +\beta\{\varphi(X, Y) \eta(Z)-\varphi(X, Z) \eta(Y)\}
\end{aligned}
$$

for certain smooth functions $\alpha$ and $\beta$ on $M^{2 n+1}$ and for all tangetn vectors $X, Y, Z \in T M^{2 n+1}$, where $\nabla$ means the covariant differentiation with respect to $g$. In that case we will say that a trans-Sasakian structure is of type $(\alpha, \beta)$ or of an $(\alpha, \beta)$-type, [5].

Remark 1.1. A $(-1,0)$-type (resp. (0,1)-type) trans-Sasakian manifold is a Sasakian (resp. a Kenmotsu) manifold. 
In a trans-Sasakian manifold of $(\alpha, \beta)$-type, we know, [5], that

$$
\begin{aligned}
& \nabla_{X} \xi=-\alpha \varphi X+\beta\{X-\eta(X) \xi\}, \\
&\left(\nabla_{X} \eta\right)(Y)=- \alpha g(\varphi X, Y)+\beta g(\varphi X, \varphi Y), \\
& R(X, Y, Z, \xi)=(X \alpha) g(\varphi Y, Z)-(Y \alpha) g(\varphi X, Z) \quad-(X \beta) g(\varphi Y, \varphi Z)+(Y \beta) g(\varphi X, \varphi Z) \\
&+\left(\alpha^{2}-\beta^{2}\right) A(X, Y, Z)-2 \alpha \beta A(X, Y, \varphi Z), \\
& \rho(X, \xi)=\left\{2 n\left(\alpha^{2}-\beta^{2}\right)-(\xi \beta)\right\} \eta(X)-\alpha(\varphi X)-(2 n-1)(X \beta)
\end{aligned}
$$

for any $X, Y \in T M^{2 n+1}$, where $\rho$ is the Ricci tensor with respect to $g$ and $A(X, Y, Z)$ is defined as

$$
A(X, Y, Z)=g(Z, Y) \eta(X)-g(Z, X) \eta(Y)
$$

for any $X, Y, Z \in T M^{2 n+1}$.

The following equations (1.7), (1.8), (1.9), (1.10) and (1.11) are very useful for calculations of the $(\mathrm{CHR})_{3}$-curvature tensor in a trans-Sasakian manifold.

By virtue of (1.4) and the Bianci identity, we have

$$
\begin{gathered}
-R(X, Y, Z, \varphi W)+R(X, Y, W, \varphi Z)= \\
=(X \alpha) A(Z, W, Y)-(Y \alpha) A(Z, W, X)+ \\
\quad+(X \beta) A(Z, W, \varphi Y)-(Y \beta) A(Z, W, \varphi X)+ \\
+\left(\alpha^{2}-\beta^{2}\right)\{g(Y, W) g(\varphi X, Z)-g(Y, Z) g(\varphi X, W)- \\
\quad-g(X, W) g(\varphi Y, Z)+g(X, Z) g(\varphi Y, W)\}+ \\
+2 \alpha \beta\{g(\varphi X, W) g(\varphi Y, Z)-g(\varphi Y, W) g(\varphi X, Z)+ \\
+g(X, Z) g(Y, W)-g(X, W) g(Y, Z)\}
\end{gathered}
$$

for any $X, Y, Z, W \in T M^{2 n+1}$. By virtue of (1.6), we can easily obtain

$$
\begin{aligned}
& R(\varphi X, Y, Z, \varphi W)+ R(\varphi X, Y, \varphi Z, W)= \\
&=-(\varphi X \alpha) A(Z, W, Y)+(Y \alpha) B(Z, W, X)+ \\
&+(\varphi X \beta) A(Z, W, Y)-(Y \beta) A(Z, W, X)+ \\
&+\left(\alpha^{2}-\beta^{2}\right)\{g(\varphi X, Z) g(\varphi Y, W)-g(\varphi X, W) g(\varphi Y, Z) \\
&+g(X, W) g(Y, Z)-g(X, Z) g(Y, W) \\
&+g(Y, W) \eta(X) \eta(Z)-g(Y, Z) \eta(X) \eta(W)\} \\
&+2 \alpha \beta\{g(X,W) g(\varphi Y, Z)-g(X, Z) g(\varphi Y, W) \\
&+g(Y, Z) g(\varphi X, W)-g(Y, W) g(\varphi X, Z) \\
&+g(\varphi Y, W) \eta(X) \eta(Z)-g(\varphi Y, Z) \eta(X) \eta(W),
\end{aligned}
$$


K. Matsumoto

and

$$
\begin{gathered}
R(X, Y, \varphi Z, \varphi W)=R(X, Y, Z, W)+ \\
+(X \alpha) A(Z, W, \varphi Y)-(Y \alpha) A(Z, W, \varphi X) \\
\quad-(X \beta) A(Z, W, Y)+(Y \beta) A(Z, W, X) \\
+\left(\alpha^{2}-\beta^{2}\right)\{g(Y, W) g(X, Z)-g(X, W) g(Y, Z) \\
\quad-g(\varphi X, Z) g(\varphi Y, W)+g(\varphi X, W) g(\varphi Y, Z)\} \\
+2 \alpha \beta\{g(\varphi Y, W) g(X, Z)-g(\varphi X, W) g(Y, Z) \\
\quad-g(\varphi Y, Z) g(X, W)+g(\varphi X, Z) g(Y, W)\}
\end{gathered}
$$

for any $X, Y, Z, W \in T M^{2 n+1}$.

Moreover, we have from the above equation

$$
\begin{gathered}
R(\varphi X, \varphi Y, \varphi Z, \varphi W)=R(X, Y, Z, W) \\
+(Z \alpha) A(X, Y, \varphi W)-(W \alpha) A(X, Y, \varphi Z) \\
-(Z \beta) A(X, Y, W)+(W \beta) A(X, Y, Z) \\
-(\varphi X \alpha) A(Z, W, Y)+(\varphi Y \alpha) A(Z, W, X) \\
-(\varphi X \beta) A(Z, W, \varphi Y)+(\varphi Y \beta) A(Z, W, \varphi X) \\
+\left(\alpha^{2}-\beta^{2}\right)\{A(X, Y, W) \eta(Z)-A(X, Y, Z) \eta(W)\} \\
+2 \alpha \beta[2\{g(X, W) g(\varphi Y, Z)-g(X, Z) g(\varphi Y, W) \\
+g(Y, W) g(\varphi X, Z)-g(Y, Z) g(\varphi X, W)\} \\
-A(X, Y, \varphi W) \eta(Z)+A(X, Y, \varphi Z) \eta(W)]
\end{gathered}
$$

for any $X, Y, Z, W \in T M^{2 n+1}$.

By virtue of $R(X, Y, Z, W)=R(Z, W, X, Y)$ for any $X, Y, Z, W \in T M^{2 n+1}$, we have from (1.10)

$$
\begin{gathered}
\{(X \alpha)+(\varphi X \beta)\} A(Z, W, \varphi Y)-\{(Y \alpha)+(\varphi Y \beta)\} A(Z, W, \varphi X) \\
-\{(Z \alpha)+(\varphi Z \beta)\} A(X, Y, \varphi W)+\{(W \alpha)+(\varphi W \beta)\} A(X, Y, \varphi Z) \\
-\{(X \beta)-(\varphi X \alpha)\} A(Z, W, Y)+\{(Y \beta)-(\varphi Y \alpha)\} A(Z, W, X) \\
+\{(Z \beta)-(\varphi Z \alpha)\} A(X, Y, W)-\{(W \beta)-(\varphi W \alpha)\} A(X, Y, Z) \\
=4 \alpha \beta[2\{g(X, W) g(\varphi Y, Z)-g(X, Z) g(\varphi Y, W) \\
+g(Y, Z) g(\varphi X, W)-g(Y, W) g(\varphi X, Z)\} \\
+A(Z, W, \varphi Y) \eta(X)-A(Z, W, \varphi X) \eta(Y)]
\end{gathered}
$$

for any $X, Y, Z, W \in T M^{2 n+1}$. Thus we have 
Proposition 1.2. In an $(\alpha, \beta)$-type trans-Sasakian manifold $M^{2 n+1}$, the functions $\alpha$ and $\beta$ satisfy (1.11).

\section{2. $(\mathrm{CHR})_{3}$-CURVATURE TENSOR IN A TRANS-SASAKIAN MANIFOLD}

In this section, we consider the $(C H R)_{3}$-curvature tensor in a transSasakian manifold.

The $(\mathrm{CHR})_{3}$-curvature tensor in an almost contact Riemannian manifold is defined by

$$
\begin{aligned}
& 16(C H R)_{3}(X, Y, Z, W)= \\
&=3\{ R(X, Y, Z, W)+R(\varphi X, \varphi Y, Z, W) \\
&+R(X, Y, \varphi Z, \varphi W)+R(\varphi X, \varphi Y, \varphi Z, \varphi W)\} \\
&-R(X, Z, \varphi W, \varphi Y)-R(\varphi X, \varphi Z, W, Y)-R(X, W, \varphi Y, \varphi Z)-R(\varphi X, \varphi W, Y, Z) \\
&+R(\varphi X, Z, \varphi W, Y)+R(X, \varphi Z, W, \varphi Y) \\
&+R(\varphi X, W, Y, \varphi Z)+R(X, \varphi W, \varphi Y, Z) \\
&+\eta( P(Z, W, Y)-\eta(Y) P(Z, W, X) \\
&+\eta(Z) P(X, Y, W)-\eta(W) P(X, Y, Z) \\
&+\eta(X) \eta(W) Q(Y, Z)-\eta(X) \eta(Z) Q(Y, W) \\
&+\eta(Y) \eta(Z) Q(W, X)-\eta(Y) \eta(W) Q(Z, X),
\end{aligned}
$$

where we put

$$
\begin{aligned}
P(X, Y, Z) & =3\{R(X, Y, Z, \xi)+R(\varphi X, \varphi Y, Z, \xi)\}+R(\varphi X, \varphi Z, Y, \xi) \\
& +R(\varphi Z, \varphi Y, X, \xi)-R(X, \varphi Z, \varphi Y, \xi)-R(\varphi Z, Y, \varphi X, \xi)
\end{aligned}
$$

and

$$
Q(X, Y)=3 R(\xi, X, Y, \xi)-R(\xi, \varphi X, \varphi Y, \xi) .
$$

for any $X, Y, Z \in T M^{2 n+1}$, [3]. We call this tensor field a contact holomorphic Riemannian curvature tensor or briefly $(\mathrm{CHR})_{3}$-curvature tensor in an almost contact Riemannian manifold. Hereafter, we assume that all vector fields are elements of $T M^{2 n+1}$.

Now, to calculate $(\mathrm{CHR})_{3}$-curvature tensor in a trans-Sasakian manifold $M^{2 n+1}$, we separate this tensor field as the following 5-parts:

$$
\begin{aligned}
& R(X, Y, Z, W)+R(\varphi X, \varphi Y, Z, W)+ \\
& \quad+R(X, Y, \varphi Z, \varphi W)+R(\varphi X, \varphi Y, \varphi Z, \varphi W), \\
& R(X, Z, \varphi W, \varphi Y)+R(\varphi X, \varphi Z, W, Y)+ \\
& \quad+R(X, W, \varphi Y, \varphi Z)+R(\varphi X, \varphi W, Y, Z),
\end{aligned}
$$


(III)

$$
\begin{aligned}
& R(\varphi X, Z, \varphi W, Y)+R(X, \varphi Z, W, \varphi Y)+ \\
& +R(\varphi X, W, Y, \varphi Z)+R(X, \varphi W, \varphi Y, Z), \\
& \text { (IV) } \quad \eta(X) P(Z, W, Y)-\eta(Y) P(Z, W, X)+ \\
& +\eta(Z) P(X, Y, W)-\eta(W) P(X, Y, Z), \\
& \text { (V) } \quad \eta(X) \eta(W) Q(Y, Z)-\eta(X) \eta(Z) Q(Y, W)+ \\
& +\eta(Y) \eta(Z) Q(W, X)-\eta(Y) \eta(W) Q(Z, X) .
\end{aligned}
$$

Then we know that

$$
16(C H R)_{3}(X, Y, Z, W)=3(\mathrm{I})-(\mathrm{II})+(\mathrm{III})+(\mathrm{IV})+(\mathrm{V}) .
$$

Using (1.9) and (1.10) we get that

$$
\begin{gathered}
(\mathrm{I})=4 R(X, Y, Z, W)+ \\
+\{(X \alpha)-(\varphi X \beta)\} A(Z, W, \varphi Y)-\{(Y \alpha)-(\varphi Y \beta)\} A(Z, W, \varphi X) \\
+2(Z \alpha) A(X, Y, \varphi W)-2(W \alpha) A(X, Y, \varphi Z)- \\
-\{(X \beta)+(\varphi X \alpha)\} A(Z, W, Y)+\{(Y \beta)+(\varphi Y \alpha)\} A(Z, W, X) \\
-2(Z \beta) A(X, Y, W)+2(W \beta) A(X, Y, Z) \\
+\left(\alpha^{2}-\beta^{2}\right)[2\{g(X, Z) g(Y, W)-g(X, W) g(Y, Z)+ \\
\quad+g(\varphi X, W) g(\varphi Y, Z)-g(\varphi X, Z) g(\varphi Y, W)\} \\
\quad+A(Z, W, Y) \eta(X)-A(Z, W, X) \eta(Y)] \\
+2 \alpha \beta[2\{g(Y, Z) g(\varphi X, W)+g(X, Z) g(\varphi Y, W) \\
\quad+g(X, W) g(\varphi Y, Z)-g(Y, W) g(\varphi X, Z)\} \\
\quad+A(X, Y, \varphi Z) \eta(W)-A(X, Y, \varphi W) \eta(Z)] .
\end{gathered}
$$

Using (1.9), we obtain 


$$
\begin{gathered}
-(\mathrm{II})=2 R(X, Y, Z, W)+(X \alpha)\{A(Z, Y, \varphi W)-A(W, Y, \varphi Z)\} \\
-(Y \alpha)\{A(Z, X, \varphi W)-A(W, X, \varphi Z)\} \\
+(Z \alpha)\{A(X, W, \varphi Y)-A(Y, W, \varphi X)\} \\
-(W \alpha)\{A(X, Z, \varphi Y)-A(Y, Z, \varphi X)\} \\
-(X \beta)\{A(Z, Y, W)-A(W, Y, Z)\} \\
+(Y \beta)\{A(Z, X, W)-A(W, X, Z)\} \\
-(Z \beta)\{A(X, W, Y)-A(Y, W, X)\} \\
+(W \beta)\{A(X, Z, Y)-A(Y, Z, X)\} \\
+2\left(\alpha^{2}-\beta^{2}\right)\{g(X, Z) g(Y, W)-g(X, W) g(Y, Z) \\
-g(\varphi X, W) g(\varphi Y, Z)+g(\varphi X, Z) g(\varphi Y, W) \\
-2 g(\varphi X, Y) g(\varphi Z, W)\} .
\end{gathered}
$$

(III) is separated as $(A)+(B)$, where we put

$$
\begin{aligned}
(A)= & R(\varphi X, Z, \varphi W, Y)+R(\varphi X, W, Y, \varphi Z) \\
=- & \{R(\varphi X, \varphi W, Y, Z)+R(\varphi X \varphi Z, W, Y)\} \\
& -\{R(\varphi X, Y, Z, \varphi W)+R(\varphi X, Y, \varphi Z, W)\}, \\
(B)= & R(X, \varphi Z, W, \varphi Y)+R(X, \varphi W, \varphi Y, Z) .
\end{aligned}
$$

By virtue of (1.9), we obtain

$$
\begin{gathered}
-\{R(\varphi X, \varphi W, Y, Z)+R(\varphi X \varphi Z, W, Y)\}=R(Z, Y, Z, W)+ \\
+(Y \alpha)\{A(X, Z, \varphi W)-A(X, W, \varphi Z)\}+ \\
+(Z \alpha) A(X, W, \varphi Y)-(W \alpha) A(X, Z, \varphi Y)+ \\
+(Y \beta)\{A(X, W, Z)-A(X, Z, W)\}- \\
-(Z \beta) A(X, W, Y)+(W \beta) A(X, Z, Y) \\
+\left(\alpha^{2}-\beta^{2}\right)\{g(X, Z) g(Y, W)-g(X, W) g(Y, Z) \\
+g(\varphi X, Z) g(\varphi Y, W)-g(\varphi X, W) g(\varphi Y, Z) \\
-2 g(\varphi X, Y) g(\varphi Z, W)\} \\
+2 \alpha \beta\{g(X, Z) g(\varphi Y, W)-g(Y, W) g(\varphi X, Z) \\
+g(Y, Z) g(\varphi X, W)-g(X, W) g(\varphi Y, Z) \\
-2 g(X, Y) g(\varphi Z, W)\} .
\end{gathered}
$$

Thus we have from (2.5) and (2.6) 


$$
\begin{aligned}
(A)=R(X, Y, Z, W)+ & \\
+ & +\varphi X) A(Z, W, Y)-2(Y \alpha) g(\varphi Z, W) \eta(X) \\
& +(Z \alpha) A(X, W, \varphi Y)-(W \alpha) A(X, Z, \varphi Y) \\
& +(\varphi X \beta) A(Z, W, \varphi Y)+2(Y \beta) A(Z, W, X) \\
& \quad-(Z \beta) A(X, W, Y)+(W \beta) A(X, Z, Y) \\
+ & \left(\alpha^{2}-\beta^{2}\right)[2\{g(X, Z) g(Y, W)-g(X, W) g(Y, Z)\} \\
& \quad-A(Z, W, Y) \eta(X)] \\
+ & 2 \alpha \beta[2\{g(X, Z) g(\varphi Y, W)-g(X, W) g(\varphi Y, Z) \\
& -g(X, Y) g(\varphi Z, W)\}-A(Z, W, \varphi Y) \eta(X)] .
\end{aligned}
$$

Since, (B) is the equation which change $X \Leftrightarrow Y$ and $Z \Leftrightarrow W$ in (A), we have

$$
\begin{gathered}
(B)=R(X, Y, Z, W)+(\varphi Y \alpha) A(W, Z, X)-2(X \alpha) g(\varphi W, Z) \eta(Y) \\
+(W \alpha) A(Y, Z, \varphi X)-(Z \alpha) A(Y, W, \varphi X) \\
+(\varphi Y \beta) A(W, Z, \varphi X)+2(X \beta) A(W, Z, Y) \\
\quad-(W \beta) A(Y, Z, X)+(Z \beta) A(Y, W, X) \\
+\left(\alpha^{2}-\beta^{2}\right)[2\{g(Y, W) g(X, Z)-g(Y, Z) g(X, W)\}-A(W, Z, X) \eta(Y)] \\
+2 \alpha \beta[2\{g(Y, W) g(\varphi X, Z)-g(Y, Z) g(\varphi X, W) \\
\quad-g(X, Y) g(\varphi W, Z)\}-A(W, Z, \varphi X) \eta(Y)] .
\end{gathered}
$$

By virtue of the above two equations, we obtain

$$
\begin{gathered}
(\mathrm{III})=2 R(X, Y, Z, W)+(\varphi X \alpha) A(Z, W, Y)-(\varphi Y \alpha) A(W, Z, X) \\
+2(X \alpha) g(\varphi Z, W) \eta(Y)-2(Y \alpha) g(\varphi Z, W) \eta(X) \\
-(Z \alpha)\{A(X, Y, \varphi W)-2 g(\varphi X, Y) \eta(W)\} \\
+(W \alpha)\{A(X, Y, \varphi Z)-2 g(\varphi X, Y) \eta(Z)\} \\
+(\varphi X \beta) A(Z, W, \varphi Y)-(\varphi Y \beta) A(Z, W, \varphi X) \\
-2(X \beta) A(Z, W, Y)+2(Y \beta) A(Z, W, X) \\
-(Z \beta) A(X, Y, W)+(W \beta) A(X, Y, Z)
\end{gathered}
$$




$$
\begin{gathered}
+\left(\alpha^{2}-\beta^{2}\right)[4\{g(X, Z) g(Y, W)-g(X, W) g(Y, Z)-g(\varphi X, Y) g(\varphi Z, W)\} \\
-A(Z, W, Y) \eta(X)+A(Z, W, X) \eta(Y)] \\
+2 \alpha \beta[2\{g(X, Z) g(\varphi Y, W)-g(X, W) g(\varphi Y, Z) \\
+g(Y, W) g(\varphi X, Z)-g(Y, Z) g(\varphi X, W)\} \\
-A(Z, W, \varphi X) \eta(Y)+A(Z, W, \varphi X) \eta(Y)] .
\end{gathered}
$$

Next, to calculate (IV) in a trans-Sasakian manifold, we have to get $P(X, Y, Z)$ which defined by $(2.2)$ in a trans-Sasakian manifold. By virtue of (1.5) we obtain that

$$
\begin{aligned}
P(X, Y, Z) & =4\{(X \alpha) g(\varphi Y, Z)-(Y \alpha) g(\varphi X, Z)\} \\
& -2\{(X \beta) g(\varphi Y, \varphi Z)-(Y \beta) g(\varphi X, \varphi Z)\} \\
& -4\{(\varphi X \alpha) g(\varphi Y, \varphi Z)-(\varphi Y \alpha) g(\varphi X, \varphi Z)\} \\
& -2\{(\varphi X \beta) g(\varphi Y, Z)-(\varphi Y \beta) g(\varphi X, Z)\} \\
& +4(\varphi Z \beta) g(\varphi X, Y)+2\left(\alpha^{2}-\beta^{2}\right) A(X, Y, Z)-8 \alpha \beta A(X, Y, \varphi Z) .
\end{aligned}
$$

Using the above equation, we get

$$
\begin{aligned}
(\mathrm{IV}) & =2\{2(X \alpha)-(\varphi X \beta)\} A(Z, W, \varphi Y)-2\{2(Y \alpha)-(\varphi Y \beta)\} A(Z, W, \varphi X) \\
& +2\{2(Z \alpha)-(\varphi Z \beta)\} A(X, Y, \varphi W)-2\{2(W \alpha)-(\varphi W \beta)\} A(X, Y, \varphi Z) \\
& -2\{2(\varphi X \alpha)+(X \beta)\} A(Z, W, Y)+2\{2(\varphi Y \alpha)+(Y \beta)\} A(Z, W, X) \\
& -2\{2(\varphi Z \alpha)+(Z \beta)\} A(X, Y, W)+2\{2(\varphi W \alpha)+(W \beta)\} A(X, Y, Z) \\
& +4\{(\varphi Y \beta) g(\varphi Z, W) \eta(X)-(\varphi X \beta) g(\varphi Z, W) \eta(Y) \\
& \quad+(\varphi W \beta) g(\varphi X, Y) \eta(Z)-(\varphi Z \beta) g(\varphi X, Y) \eta(W)\} \\
& +4\left(\alpha^{2}-\beta^{2}\right)\{A(Z, W, Y) \eta(X)-A(Z, W, X) \eta(Y)\} .
\end{aligned}
$$

Finally, we calculate (V) in a trans-Sasakian manifold.

By virtue of $(1.5)_{3}$, we have

$$
\begin{aligned}
R(\xi, X, Y, \xi)=\{ & (\xi \alpha)+2 \alpha \beta\} g(\varphi X, Y)+ \\
& +\left\{\left(\alpha^{2}-\beta^{2}\right)-(\xi \beta)\right\} g(\varphi X, \varphi Y)
\end{aligned}
$$

In (2.7), the left hand side is symmetric with respect to $X$ and $Y$. So we have 
Proposition 2.1. In a trans-Sasakian manifold, the condition

$$
(\xi \alpha)+2 \alpha \beta=0
$$

holds.

Thus, (2.7) is written as

$$
R(\xi, X, Y, \xi)=\left\{\left(\alpha^{2}-\beta^{2}\right)-(\xi \beta)\right\} g(\varphi X, \varphi Y) .
$$

By virtue of (2.8), we can easily obtain that

$$
R(\xi, X, Y, \xi)=R(\xi, \varphi X, \varphi Y, \xi) .
$$

Thus we have from the above equation

$$
Q(X, Y)=2\left\{\left(\alpha^{2}-\beta^{2}\right)-(\xi \beta)\right\} g(\varphi X, \varphi Y) .
$$

Thus we have from (2.9)

$$
\left.(\mathrm{V})=-2\left\{\left(\alpha^{2}-\beta^{2}\right)-(\xi \beta)\right\}\{A(Z, W, Y) \eta(X)-A(Z, W, X) \eta Y)\right\} .
$$

By virtue of (1.11), (I), (II), (III), (IV) and (V), the $(C H R)_{3}$-curvature tensor in a trans-Sasakian manifold is written as follows:

$$
\begin{aligned}
& 16(C H R)_{3}(X, Y, Z, W)=16 R(X, Y, Z, W) \\
& +(X \alpha)\{7 A(Z, W, \varphi Y)+4 g(\varphi Z, W) \eta(Y)\} \\
& \text { - }(Y \alpha)\{7 A(Z, W, \varphi X)+4 g(\varphi Z, W) \eta(X)\} \\
& +(Z \alpha)\{7 A(X, Y, \varphi W)+4 g(\varphi X, Y) \eta(W)\} \\
& \text { - }(W \alpha)\{7 A(X, Y, \varphi Z)+4 g(\varphi X, Y) \eta(Z)\} \\
& -5\{(\varphi X \alpha) A(Z, W, Y)-(\varphi Y \alpha) A(Z, W, X) \\
& +(\varphi Z \alpha) A(X, Y, W)-(\varphi W \alpha) A(X, Y, Z)\} \\
& -9\{(X \beta) A(Z, W, Y)-(Y \beta) A(Z, W, X) \\
& +(Z \beta) A(X, Y, W)-(W \beta) A(X, Y, Z)\} \\
& -(\varphi X \beta)\{3 A(Z, W, \varphi Y)+4 g(\varphi Z, W) \eta(Y)\} \\
& +(\varphi Y \beta)\{3 A(Z, W, \varphi X)+4 g(\varphi Z, W) \eta(X)\} \\
& -(\varphi Z \beta)\{3 A(X, Y, \varphi W)+4 g(\varphi X, Y) \eta(W)\} \\
& +(\varphi W \beta)\{3 A(X, Y, \varphi Z)+4 g(\varphi X, Y) \eta(Z)\} \\
& +\left(\alpha^{2}-\beta^{2}\right)[12\{g(X, Z) g(Y, W)-g(X, W) g(Y, Z)\} \\
& +4\{g(\varphi X, W) g(\varphi Y, Z)-g(\varphi X, Z) g(\varphi Y, W) \\
& -2 g(\varphi X, Y) g(\varphi Z, W)\}] \\
& +2(\xi \beta)\{A(Z, W, Y) \eta(X)-A(Z, W, X) \eta(Y)\} .
\end{aligned}
$$


From the above equation, we can easily obtain the $(\mathrm{CHR})_{3}$-Ricci tensor $\rho_{3}$ and the $(\mathrm{CHR})_{3}$-scalar curvature $\tau_{3}$ as

$$
\begin{aligned}
8 \rho_{3}(X, Y) & =8 \rho(X, Y)+(5 n+3)[\{(\varphi X) \alpha\} \eta(Y)+\{(\varphi Y) \alpha\} \eta(X)] \\
& +(9 n-1)\{(X \beta) \eta(Y)+(Y \beta) \eta(X)\} \\
& -4\left(\alpha^{2}-\beta^{2}\right)\{(3 n-1) g(X, Y)+(n+1) \eta(X) \eta(Y)\} \\
& +2(\xi \beta)\{4 g(X, Y)-(n+3) \eta(X) \eta(Y)\} .
\end{aligned}
$$

and

$$
\tau_{3}=\tau-(3 n+1) n\left(\alpha^{2}-\beta^{2}\right)+4 n(\xi \beta),
$$

where $\tau$ denotes the scalar curvature with respect to $g$.

By virtue of $(1.5)_{4}$ and (2.11), we easily have

$$
\left.8 \rho_{3}(X, \xi)=5(n-1)\{(\varphi X) \alpha)-7(n-1)\{(X \beta)-(\xi \beta) \eta(X)\}\right\} .
$$

\section{3. $(\mathrm{CHR})_{3}$-FLAT TRANS-SASAKIAN MANiFOLDS}

An almost contact Riemannian manifold is called $(\mathrm{CHR})_{3}$-flat if the $(\mathrm{CHR})_{3}$-curvature tensor equals to zero on $M^{2 n+1}$.

Let us consider a $(\mathrm{CHR})_{3}$-flat trans-Sasakian manifold. Then the left hand side of (2.10) is zero.

Moreover, if the $(\mathrm{CHR})_{3}$-curvature tensor is flat, then the $(\mathrm{CHR})_{3}$-Ricci tensor and the $(\mathrm{CHR})_{3}$-scalar are flat. So, by virtue of (2.11) and (2.12), we respectively have

$$
\begin{aligned}
& 8 \rho(X, Y)+(5 n+3)[\{(\varphi X) \alpha\} \eta(Y)+\{(\varphi Y) \alpha\} \eta(X)]+ \\
& +(9 n-1)\{(X \beta) \eta(Y)+(Y \beta) \eta(X)\}- \\
& -4\left(\alpha^{2}-\beta^{2}\right)\{(3 n-1) g(X, Y)+(n+1) \eta(X) \eta(Y)\}+ \\
& +2(\xi \beta)\{4 g(X, Y)-(n+3) \eta(X) \eta(Y)\}=0
\end{aligned}
$$

and

$$
\tau-(3 n+1) n\left(\alpha^{2}-\beta^{2}\right)+4 n(\xi \beta)=0 .
$$

We know from (2.13)

$$
5\{(\varphi X) \alpha\}-7\{(X \beta)-(\xi \beta) \eta(X)\}=0 .
$$

From the above equation, we get

$$
\begin{aligned}
&\{(\varphi X) \alpha\} \eta(Y)+\{(\varphi Y) \alpha\} \eta(X)= \\
&+\frac{7}{5}\{(X \beta) \eta(Y)+(Y \beta) \eta(X)\}-\frac{14}{5}(\xi \beta) \eta(X) \eta(Y) .
\end{aligned}
$$


Substituting (3.4) into (3.1), we get

$$
\begin{aligned}
\rho(X, Y) & =\frac{(3 n-1)\left(\alpha^{2}-\beta^{2}\right)-2(\xi \beta)}{2} g(X, Y) \\
& +\frac{1}{2}\left\{\frac{2(10 n+9)}{5}(\xi \beta)+(n+1)\left(\alpha^{2}-\beta^{2}\right)\right\} \eta(X) \eta(Y) \\
& -\frac{2(5 n+1)}{5}\{d \beta(X) \eta(X)+d \beta(Y) \eta(X)\} .
\end{aligned}
$$

Thus we have

Theorem 3.1. A $(\mathrm{CHR})_{3}$-flat trans-Sasakian manifold is a generalized $\eta$-Einstein manifold.

Remark 3.2. The notion of a generalized $\eta$-Einstein manifold is defined by A. A. Shaikh and Y. Matsuyama, [5]. Moreover, M. C. Chaki called this manifold a generalized quasi-Einstein manifold, [1], [2].

From the above theorem, we can easily obtain

Corollary 3.3. $A(C H R)_{3}$-flat trans Sasakian manifold is $\eta$-Einstein if and only if the function $\beta$ is constant. Then the Ricci tensor $\rho$ and the scalar curvature $\tau$ with respect to $g$ are written as

$$
\rho(X, Y)=\left(\alpha^{2}-\beta^{2}\right)\left\{\frac{3 n-1}{2} g(X, Y)+\frac{n+1}{2} \eta(X) \eta(Y)\right\}
$$

and

$$
\tau=-(3 n+1) n\left(\alpha^{2}-\beta^{2}\right) .
$$

By virtue of Remark 1.1 and the above corollary, we get

Corollary 3.4. In a $(\mathrm{CHR})_{3}$-flat Sasakian, resp. Kenmotsu, manifold, the Ricci tensor $\rho$ and the scalar curvature $\tau$ with respect to $g$ satisfy

$$
\rho(X, Y)=\frac{3 n-1}{2} g(X, Y)+\frac{n+1}{2} \eta(X) \eta(Y),
$$

resp.

and

$$
\rho(X, Y)=-\left\{\frac{3 n-1}{2} g(X, Y)+\frac{n+1}{2} \eta(X) \eta(Y)\right\}
$$

$$
\tau=-(3 n+1) n \quad(\text { resp. } \quad \tau=(3 n+1) n) .
$$

Now, from (3.3), we obtain

$$
\begin{aligned}
& -5\{(\varphi X) \alpha\} A(Z, W, Y)+5\{(\varphi Y) \alpha\} A(Z, W, X)- \\
& \quad-5\{(\varphi Z) \alpha\} A(X, Y, W)+5\{(\varphi W) \alpha\} A(X, Y, Z)- \\
& -9\{(X \beta) A(Z, W, Y)-(Y \beta) A(Z, W, X)+
\end{aligned}
$$




$$
\begin{aligned}
+ & (Z \beta) A(X, Y, W)-(W \beta) A(X, Y, Z)\}= \\
=-16 & \{(X \beta) A(Z, W, Y)-(Y \beta) A(Z, W, X)+ \\
+ & (Z \beta) A(X, Y, W)-(W \beta) A(X, Y, Z)\}+ \\
& +14(\xi \beta)\{A(Z, W, Y) \eta(X)-A(Z, W, X) \eta(Y)\} .
\end{aligned}
$$

From (3.3), we get

$$
\{(\varphi X) \beta\}=-\frac{5}{7}(X \alpha)+\frac{5}{7}(\xi \alpha) \eta(X)
$$

From this, we have

$$
\begin{aligned}
& -3[\{(\varphi X) \beta\} A(Z, W, \varphi Y)-\{(\varphi Y) \beta\} A(Z, W, \varphi X)+ \\
& \quad+\{(\varphi Z) \beta\} A(X, Y, \varphi W)-\{(\varphi W) \beta\} A(X, Y, \varphi Z)]+ \\
& +7\{(X \alpha) A(Z, W, \varphi Y)-(Y \alpha) A(Z, W, \varphi X)+ \\
& \quad+(Z \alpha) A(X, Y, \varphi W)-(W \alpha) A(X, Y, \varphi Z)\}= \\
& =\frac{64}{7}\{(X \alpha) A(Z, W, \varphi Y)-(Y \alpha) A(Z, W, \varphi X)+ \\
& \quad+(Z \alpha) A(X, Y, \varphi W)-(W \alpha) A(X, Y, \varphi Z)\} .
\end{aligned}
$$

Next, since we have

$$
4[(X \alpha)-\{(\varphi X) \beta\}] g(\varphi Z, W) \eta(Y)=\frac{48}{7}(X \alpha)-\frac{20}{7}(\xi \alpha) g(\varphi Z, W) \eta(X) \eta(Y)
$$
we obtain

$$
\begin{aligned}
& 4[(X \alpha)-\{(\varphi X) \beta\}] g(\varphi Z, W) \eta(Y)- \\
& -4[(Y \alpha)-\{(\varphi Y) \beta\}] g(\varphi Z, W) \eta(X)+ \\
& +4[(Z \alpha)-\{(\varphi Z) \beta\}] g(\varphi X, Y) \eta(W)- \\
& -4[(W \alpha)-\{(\varphi W) \beta\}] g(\varphi X, Y) \eta(Z)= \\
& =\frac{48}{7}\{(X \alpha) g(\varphi Z, W) \eta(Y)-(Y \alpha) g(\varphi Z, W) \eta(X)+ \\
& +(Z \alpha) g(\varphi X, Y) \eta(W)-(W \alpha) g(\varphi X, Y) \eta(Z)\} .
\end{aligned}
$$


Using (3.6), (3.7) and (3.8), the curvature tensor $R$ with respect to $g$ is written as

$$
\begin{aligned}
R(X, Y, Z, W)=\frac{1}{7}[(X \alpha)\{4 A(W, Z, \varphi Y)+3 g(\varphi W, Z), \eta(Y)\} \\
-(Y \alpha)\{4 A(W, Z, \varphi X)+3 g(\varphi W, Z) \eta(X)\} \\
+(Z \alpha)\{4 A(Y, X, \varphi W)+3 g(\varphi Y, X) \eta(W)\} \\
\quad-(W \alpha)\{4 A(Y, X, \varphi Z)+3 g(\varphi Y, X) \eta(Z)\}] \\
+(X \beta) A(W, Z, Y)-(Y \beta) A(W, Z, X) \\
\quad-(Z \beta) A(Y, X, W)+(W \beta) A(Y, X, Z) \\
+\frac{1}{4}\left(\alpha^{2}-\beta^{2}\right)[3\{g(X, W) g(Y, Z)-g(X, Z) g(Y, W)\} \\
\quad-g(\varphi X, W) g(\varphi Y, Z)+g(\varphi X, Z) g(\varphi Y, W) \\
\quad+2 g(\varphi X, Y) g(\varphi Z, W)] \\
-\frac{1}{4}\left\{\left(\alpha^{2}-\beta^{2}\right)-(\xi \beta)\right\}\{A(Z, W, Y) \eta(X)-A(Z, W, X) \eta(Y)\} .
\end{aligned}
$$

Thus we get

Theorem 3.5. If a trans-Sasakian manifold is $(\mathrm{CHR})_{3}$-flat, the the curvature tensor satisfies (3.9).

By virtue of Remark 1.1 and the above theorem, we have

Corollary 3.6. In a $(\mathrm{CHR})_{3}$-flat Sasakian, resp. Kenmotsu, manifold, the curvature tensor $R$ with respect to $g$ are written by

$$
\begin{aligned}
R(X, Y, Z, W)=\frac{1}{4}[3\{g(X, W) g(Y, Z)-g(X, Z) g(Y, W)\} \\
\quad-g(\varphi X, W) g(\varphi Y, Z)+g(\varphi X, Z) g(\varphi Y, W)+2 g(\varphi X, Y) g(\varphi Z, W)] \\
\quad-\frac{1}{4}\{A(Z, W, Y) \eta(X)-A(Z, W, X) \eta(Y)\}
\end{aligned}
$$

resp.

$$
\begin{aligned}
& R(X, Y, Z, W)=-\frac{1}{4}[3\{g(X, W) g(Y, Z)-g(X, Z) g(Y, W)\} \\
& \quad-g(\varphi X, W) g(\varphi Y, Z)+g(\varphi X, Z) g(\varphi Y, W)+2 g(\varphi X, Y) g(\varphi Z, W)] \\
& \quad+\frac{1}{4}\{A(Z, W, Y) \eta(X)-A(Z, W, X) \eta(Y)\} .
\end{aligned}
$$


Remark 3.7. The above corollary shows that a $(\mathrm{CHR})_{3}$-flat Sasakian (resp. Kenmotsu) manifold is a Sasakian (resp. Kenmotsu) space form with zero holomorphic sectional curvature.

Remark 3.8. Of course, we can get (3.2) and (3.5) from Theorem 3.1 directly.

\section{ACKNOWLEDGEMENTS}

The author deeply thanks to Professor Yevhen Cherevko at Odesa National Economic University for his very kind support.

\section{REFERENCES}

[1] M. C. Chaki. On generalized quasi Einstein manifolds. Publ. Math. Debrecen, 58(4):683691, 2001.

[2] M. C. Chaki, R. K. Maity. On quasi Einstein manifolds. Publ. Math. Debrecen, 57(34):297-306, 2000.

[3] Koji Matsumoto. A new curvaturelike tensor field in an almost contact Riemannian manifold II. Publ. Inst. Math. (Beograd) (N.S.), 103(117):113-128, 2018, doi: $10.2298 /$ pim1817113m.

[4] Mileva Prvanović. Conformally invariant tensors of an almost Hermitian manifold associated with the holomorphic curvature tensor. J. Geom., 103(1):89-101, 2012, doi: $10.1007 / \mathrm{s} 00022-012-0111-9$.

[5] A. A. Shaikh, Y. Matsuyama. On trans-Sasakian manifolds. SUT J. Math., 45(1):25-41, 2009.

Koji Matsumoto

2-3-65 Nishi-Odori, Yonezawa, Yamagata, 992-0059, JAPAn

Email: tokiko_matsumoto@yahoo.com 